\title{
Torsion, Autoamputation, and Reimplantation of Viable Ovarian Dermoid Cyst
}

\author{
Alexis D. Greene, MD, Andrzej K. Breborowicz, MD, PhD, Anne Hardart, MD \\ Department of Obstetrics \& Gynecology, St Luke's Roosevelt Hospital Center, New York, NY, USA (all authors).
}

\begin{abstract}
Introduction: Case reports of parasitic teratomas exist; however, most viable ovarian dermoids reimplant into the omentum. It is rare to report a viable ovary containing a dermoid that has undergone autoamputation and reimplantation in the posterior cul-de-sac.
\end{abstract}

Case Description: We report the case of a 48-year-old woman with a history of lower abdominal pain that was relieved with pain control and recurred 1 year later, prompting a diagnostic laparoscopy. Laparoscopy confirmed an ovarian dermoid cyst in the posterior cul-de-sac. Intraoperatively, the ovary containing the dermoid appeared viable; this was confirmed by histopathology.

Discussion: The mass found on laparoscopy can best be explained by torsion, autoamputation, and reimplantation of the ovary containing the mature cystic teratoma into the posterior cul-de-sac.

Key Words: Dermoid cyst, Ovarian torsion, Autoamputation, Teratoma.

Citation Greene AD, Breborowicz AK, Hardart A. Torsion, autoamputation, and reimplantation of viable ovarian dermoid cyst. CRSLS e2014.00192. DOI 10.4293/CRSLS.2014.00192.

Copyright (C) 2014 SLS This is an open-access article distributed under the terms of the Creative Commons Attribution-Noncommercial-ShareAlike 3.0 Unported license, which permits unrestricted noncommercial use, distribution, and reproduction in any medium, provided the original author and source are credited.

Address correspondence to: Alexis D. Greene, MD, Department of Obstetrics \& Gynecology, St Luke's Roosevelt Hospital Center, 1000 Tenth Ave, Ste 10C, New York, NY 10019, USA. Telephone: (212) 523-4000, Fax: (212) 523-8012, E-mail: algreene@chpnet.org

\section{INTRODUCTION}

A parasitic ovary, which is an ovary that has reimplanted elsewhere in the abdomen, obtaining a new blood supply, is a rare occurrence not commonly discussed in the gynecology literature. It is usually found in neonates, and the ovary is most often reimplanted in the omentum. ${ }^{1}$ Extremely rare is the case in which a viable ovary is found in the posterior cul-de-sac in an adult. ${ }^{1-3}$ This case involves a woman with a history of abdominal pain for 1 year who was found on diagnostic laparoscopy to have a mature cystic teratoma that had undergone autoamputation with viable ovarian tissue adherent to the posterior cul-de-sac, attached to a viable fimbriated portion of the fallopian tube.

\section{CASE DESCRIPTION}

A 48-year-old woman, gravida 7 , para 5, originally presented to the emergency department 14 months before surgery with right-sided flank pain radiating to her groin. A computed tomography (CT) scan with contrast was obtained to rule out nephrolithiasis. Although no kidney stone was seen, a right- sided cystic mass consistent with hydrosalpinx was found, along with a $4 \times 3-\mathrm{cm}$ mass below the cystic structure with thick calcifications (Figure 1). This was described as a possible ovarian mass, and ultrasonographic examination was recommended. Endovaginal and transabdominal ultrasonography confirmed hydrosalpinx and identified the calcified mass as a possible leiomyoma; however, no connection to the uterus or cervix was seen. The patient was sent home from the emergency department with pain management and was advised to undergo follow-up in the gynecology clinic as an outpatient. The pain subsequently resolved, and the patient was then lost to follow-up until 1 week before surgery when her pain recurred. Pelvic ultrasonography at that time showed a persistent and slightly enlarged right-sided hydrosalpinx. The previously noted calcified mass was no longer seen. Intermittent torsion was suspected, and a diagnostic laparoscopy was performed.

On laparoscopy, a right-sided hydrosalpinx with torsion was noted and excised (Figure 2). The right ovary was not found near the tube in its proper anatomic location, and no fim- 


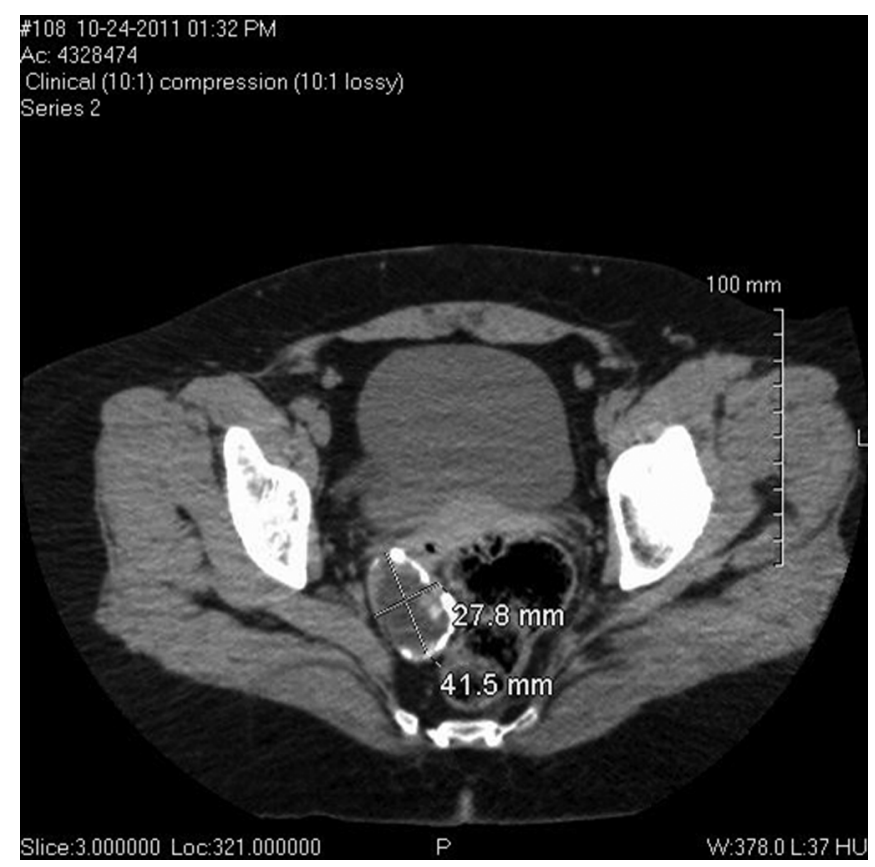

Figure 1. CT imaging of calcified mass inferoposterior to uterus.

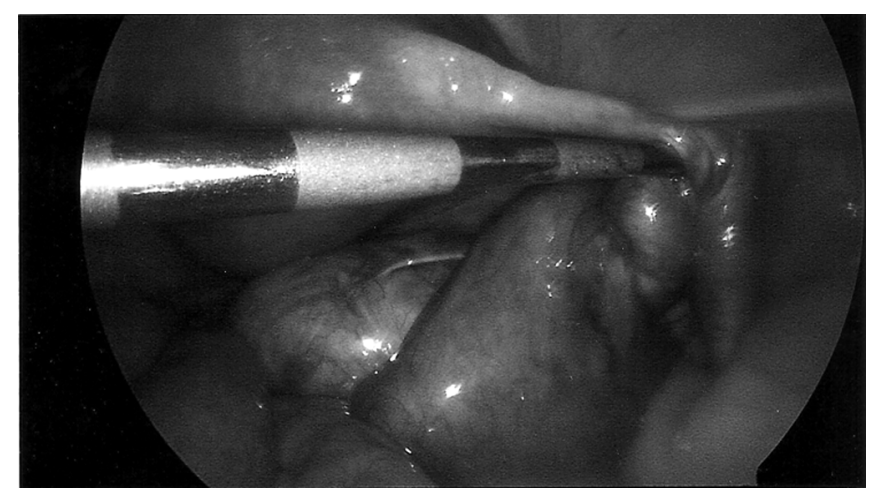

Figure 2. Laparoscopic image of torsion of right hydrosalpinx.

briae were seen on the fallopian tube. The uterus, left ovary, and tube appeared normal. In the posterior cul-de-sac, a mass measuring approximately $5 \mathrm{~cm}$ was seen. At first, it was difficult to identify what it was; it appeared to be encased in a peritoneal layer. After careful sharp and blunt dissection of the mass, it was identified as an ovary and dermoid cyst, attached to a portion of the fimbriated end of the fallopian tube. The ovarian stroma around the dermoid appeared viable, and the fimbria appeared pink and normal (Figure 3). As the ovary was dissected out from the cul-de-sac, the area was hemostatic, and the ovary did not appear connected to the uterus or other nearby pelvic structures. A rectal examination was performed throughout the dissec-

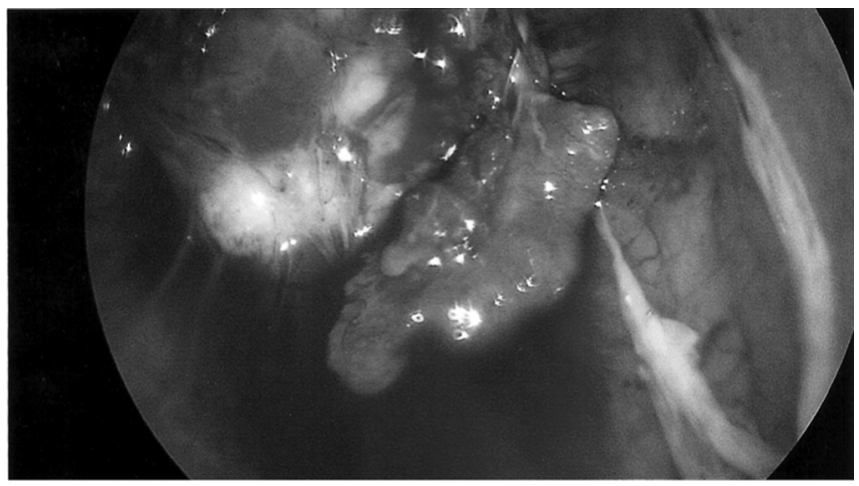

Figure 3. Laparoscopic image of posterior cul-de-sac mass after dissection.

tion to ensure that the mass was not connected to the rectum. The mass was removed, and the surgery was uncomplicated. Postoperatively, the patient returned for follow-up and her pain had resolved.

The histology slides were reviewed with the pathologist. The cul-de-sac mass was $7.3 \times 5.0 \mathrm{~cm}$, containing ovarian tissue, a mature cystic teratoma, and a fimbriated portion of the fallopian tube. Viable ovarian stromal tissue was seen with a normal blood supply on the outer layer of the specimen. The fimbria attached to the ovary was also viable. Coagulation necrosis was noted in the center of the ovary, characterized by nonviable cells without nuclei. Hair shafts and adipose tissue were identified, consistent with a degenerative, mature cystic teratoma. Hydrosalpinx of the fallopian tube with mild to moderate chronic salpingitis was reported. The histologic and laparoscopic findings were consistent with an ovary that had undergone autoamputation containing a dermoid cyst and reimplanted in the posterior cul-de-sac.

\section{DISCUSSION}

The diagnosis of a parasitic ovary containing a dermoid cyst is usually unknown before surgery. In most cases the tumor is not accurately diagnosed and is often generalized as an ovarian or abdominal mass. ${ }^{1}$ Although the preoperative diagnosis is often ovarian tumor, color Doppler flow imaging of the tumor is often inconsistent with this diagnosis because there is typically no vascular connection between the mass and the adnexum. The imaging in our patient also identified a pelvic mass on CT, and similarly, no vascular connection between the mass and the uterus or ovary was seen.

Dermoid cysts may present as calcified masses in the pelvis; however, the differential diagnosis of a calcified mass includes uterine leiomyoma, ovarian cystadenoma, and a calcified lymph node. ${ }^{4}$ In our case a calcified right-sided pelvic 
mass posterior to the uterus was seen on CT scan. At the time, it was read as a probable calcified myoma.

A review of the literature on parasitic dermoids mostly shows mature cystic teratomas within the omentum. The reason for the predilection for the omentum is because of its unique role in the intra-abdominal inflammation process. ${ }^{5}$ Ushakov et al $^{1}$ reviewed 25 cases of omental dermoids, 10 of which were proven to be parasitic omental ovarian teratomas. The incidence of parasitic dermoid cysts, as these would be named after reimplantation elsewhere in the abdomen, is $0.4 \%$ of all ovarian dermoids. ${ }^{1,5}$

Ushakov et $\mathrm{al}^{1}$ reviewed 3 proposed etiologies of omental teratomas. ${ }^{5}$ The first etiology is a primary omental teratoma. Embryologically, while the germ cells are migrating along the primitive gut to the genital ridge, they may arrest at the level of the dorsal mesentery. In this scenario, a dermoid would be seen; however, no ovarian stroma would be shown, and both ovaries would be in their normal anatomic location. This could not be the case in our patient because ovarian stroma existed with the dermoid and the right ovary was missing from its proper location.

The second theory involves a supernumerary ovary that forms within the omentum. This proposed theory has never been proven because only omental dermoids without ovarian tissue have been found. ${ }^{1}$ In our case the dermoid cyst was attached to normal ovarian stroma and fimbria of the fallopian tube, so this is unlikely to explain our case.

The third theory, which is most plausible for our patient, is autoamputation and reimplantation of the ovarian dermoid. Autoamputation occurs when the ovary undergoes torsion and then amputates from its normal position. Torsion of the adnexa usually occurs on the right side. The sigmoid colon located on the left side is thought to be protective of the twisting of the left adnexum, making right adnexal torsion more common. ${ }^{1,2,4,6}$ When the blood supply to the dermoid is cut off as a result of torsion, the tumor can undergo necrosis and actually undergo autoamputation as a result. The dermoid may reimplant in neighboring structures and develop a new blood supply.

What we believe to have occurred, which would explain our patient's abdominal pain, is as follows: The original abdominal pain that the patient had was likely the right-sided ovarian torsion. The ovary containing the dermoid cyst had undergone autoamputation and fell into the posterior cul-de-sac. When the pain recurred 2 weeks before surgery, it was likely the right-sided hydrosalpinx with torsion, a separate pathology from the original pain, yet with similar symptomatology.
Two similar cases have been reported in the literature in which an ovary that had undergone autoamputation was found with a dermoid cyst in the posterior cul-de-sac; however, both were attached to the omentum. In one case the dermoid was found during a cesarean delivery. ${ }^{3}$ The patient reported a remote history of severe abdominal pain as an adolescent that resolved but was never diagnosed. During the cesarean section, the ovary, the dermoid, and a portion of the fallopian tube were found enveloped in the omentum, from which the ovary obtained its vascular supply. It was also easily dissected out from the posterior cul-de-sac. This is more consistent with most reported parasitic ovarian dermoids. The difference in our case was that the ovary that had undergone autoamputation was not attached to the omentum; it was fixed and adherent to the posterior cul-de-sac and was unrecognizable as an ovary and teratoma until dissected out.

A second case was reported in a 24-year-old woman with an acute onset of left lower quadrant pain. ${ }^{2}$ She was not operated on until several months later, showing a dermoid with viable ovarian stroma in the posterior cul-de-sac. As in the cesarean case report, the omentum was encasing and vascularizing the ovary.

Our case is unique in identifying a parasitic ovary containing a dermoid cyst that maintained a blood supply separate from the omentum. What we learned from this case is to always look for the adnexum in its appropriate location, and if it is not found, a total abdominal survey should be performed.

\section{References:}

1. Ushakov FB, Meirow D, Prus D, Libson E, BenShushan A, Rojansky N. Parasitic ovarian dermoid tumor of the omentum-a review of the literature and a report of two new cases. Eur J Obstet Gynecol Reprod Biol. 1998;81:77-82.

2. Kusaka M, Mikuni M. Ectopic ovary: a case of autoamputated ovary with mature cystic teratoma into the cul-de-sac. $J$ Obstet Gynaecol Res. 2007;33;368-370.

3. Peitsidou A, Peitsidis P, Goumalatsos N, Papaspyrou R, Mitropoulou G, Georgoulias N. Diagnosis of an autoamputated ovary with dermoid cyst during a cesarean section. Fertil Steril. 2009;1294.e9-e12.

4. Henry LM, Rauh J, Burket R. Pelvic calcification and autoamputation of the uterine adnexa in an adolescent. $J$ Adolesc Health Care. 1998;9:225-228.

5. Shetty NS, Vallabhaneni S, Patil A, Babu MM, Baig A. Unreported location and presentation for a parasitic ovarian dermoid cyst in an indirect inguinal hernia. Hernia. 2013;17(2):263-265.

6. Peh WCG, Chu FSK, Lorentz TG. Painful right iliac fossa mass caused by a migrating left ovary. Clin Imaging. 1994;18:199-202. 